\title{
Ephrin-B3 modulates hippocampal neurogenesis and the reelin signaling pathway in a pilocarpine-induced model of epilepsy
}

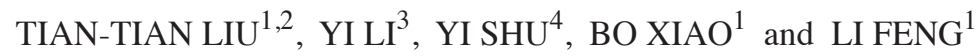 \\ ${ }^{1}$ Department of Neurology, Xiangya Hospital, Central South University, Changsha, Hunan 410008; \\ ${ }^{2}$ Department of Neurology, The First Affiliated Hospital of Zhengzhou University, Zhengzhou, Henan 450052, P.R. China; \\ ${ }^{3}$ Department of Neurology, University of Massachusetts Medical School, Worcester, MA 01604, USA; \\ ${ }^{4}$ Department of Neurology, Second Xiangya Hospital, Central South University, Changsha, Hunan 410008, P.R. China
}

Received October 9, 2017; Accepted March 5, 2018

DOI: $10.3892 /$ ijmm.2018.3543

\begin{abstract}
Ephrin-B3 is important in the regulation of cell proliferation, differentiation and migration via cell-cell contact, and can activate the reelin pathway during brain development. However, the effect of ephrin-B3 on hippocampal neurogenesis and the reelin pathway in epilepsy remains to be fully elucidated. In the present study, the expression of ephrin-B3 in pilocarpine-induced status epilepticus (SE) rats was investigated. SYBR Green-based reverse transcription-quantitative polymerase chain reaction analysis, immunohistochemical labeling and western blot analysis were used to detect the gene and protein expression levels of ephrin-B3 and reelin pathway proteins. Immunofluorescence staining of doublecortin (DCX) was utilized to analyze hippocampal neurogenesis. The data revealed that the mRNA and protein expression levels of ephrin-B3 in the hippocampus decreased during the spontaneous seizure period. Of note, the expression of reelin and its downstream phosphorylation disabled 1 (p-Dab1) were also notably decreased during the spontaneous seizure period, which showed similar dynamic changes as in the expression of ephrin-B3. In addition, it was found that the number of DCX-labeled neuronal progenitor cells was increased in the hippocampus following pilocarpine-induced SE. To further clarify the role of ephrin-B3 in neurogenesis and the reelin pathway in epilepsy, an exogenous ephrin-B3 clustering stimulator, EphB3-Fc, was infused into the bilateral hippocampus of the rats post-SE. Following EphB3-Fc injection, it was found that the expression levels of reelin and p-Dab1 were significantly increased in the epileptic rats following EphB3-Fc injection. The number of DCX-labeled neuronal progenitor
\end{abstract}

Correspondence to: Dr Bo Xiao or Dr Li Feng, Department of Neurology, Xiangya Hospital, Central South University, 87 Xiangya Road, Changsha, Hunan 410008, P.R. China

E-mail: xiaobo_xy@126.com

E-mail: fengli1982@yahoo.com.cn

Key words: epilepsy, ephrin-B3, reelin pathway, neurogenesis, neuronal excitability cells was reduced in the hippocampus of the epileptic rats. Furthermore, the intensity and frequency of spontaneous recurrent seizures and electroencephalographic seizures were attenuated in the epileptic rats post-injection. These results demonstrated the critical role of ephrin-B3 in regulation of the reelin pathway and hippocampal neurogenesis in epilepsy, providing experimental evidence that ephrin-B3 functions as a potential protective factor in epilepsy, at least in animals.

\section{Introduction}

Epilepsy is one of the most prevalent chronic neurological disorders, which affects $\sim 65,000,000$ individuals worldwide and is a global burden in terms of seizure-related disability, comorbidities and mortality rates (1). Clinically, temporal lobe epilepsy (TLE) is the most common form of drug-resistant and intractable epilepsy, and is characterized by recurrent spontaneous seizures (SRS) due to neuronal hyperactivity in the brain (2). Abnormal hippocampal neurogenesis is a prominent feature of TLE, which may contribute to the hippocampal network plasticity associated with epilepsy (3). The newborn neuron can be labeled by doublecortin (DCX), a marker of transit-amplifying cells and immature newborn neurons, which begin to have neuronal potential (4). These newborn neurons are continuously generated in the subgranular zone of the hippocampal dentate gyrus, migrate into the granule layer and terminally differentiate, mainly into new granule cell neurons (5). The newly generated ectopic granule cells have immature synapses and exhibit spontaneous discharge, which may be an important pathophysiological basis for chronic spontaneous seizures (6). However, the molecular mechanisms that regulate hippocampal neurogenesis during the spontaneous seizure period remain to be fully elucidated.

Ephrins, ligands for Eph receptors, are membrane-bound proteins which are the largest member of the receptor tyrosine kinase family and can be divided into A and B subclasses, binding either A-class or B-class ligands; ephrin ligands and receptors are important in the regulation of morphologic processes, including axon guidance, angiogenesis, cell migration and positioning, in the central nervous system (7). Increasing roles of the transmembrane ephrin-B3 ligand have been revealed in the central nervous system. In the hippocampal 
dentate gyrus, ephrin-B3/Eph-B1 is involved in several functions, including neuronal progenitor sorting, stochastic cell migration and guidance of neuronal growth cones during the early developing brain (8). Ephrin-B3 can also reduce cell death by inhibiting the functions of EphA4 receptors during adult neurogenesis in the subventricular zone of the brain (9). Based on these reports, the present study hypothesized that ephrin-B3 may affect hippocampal neurogenesis during the spontaneous seizure period following pilocarpine-induced epilepsy.

Ephrin-B3 can function by the regulation of other signaling pathways via cell-cell interactions, including the regulation of glutamate receptor signaling by inducing the tyrosine phosphorylation of NR2 subunits in glutamatergic CA1 synapses in the hippocampus (10). Of note, ephrin-B3 is involved in the activation of the reelin signaling pathway, which is essential in controlling neuronal migration in the neocortex, hippocampus and cerebellum of reelin-deficient mutant reeler mice $(11,12)$. Reelin is an extracellular protein, which affects several stages of neuronal migration and layering in the developing brain; reelin protein acts mainly by binding to low-density lipoprotein receptors and apolipoprotein $\mathrm{E}$ receptor 2, and then initiates the tyrosine phosphorylation of intracytoplasmic docking disabled protein 1 (Dab1) (13). The reelin pathway control the shapes, sizes and types of dendritic spines, and synaptic homeostasis; its dysregulation may occur in neurological and psychiatric disorders, including epilepsy, targeting hippocampal circuits (14). Although the functions of ephrin-B3 and the reelin pathway during physiological or pathological neurodevelopment are clear, the function of ephrin-B3 in modulation of the reelin pathway in epilepsy remains to be fully elucidated.

The aim of the present study was to investigate the role of ephrin-B3 in hippocampal neurogenesis and the reelin pathway in a pilocarpine-induced rat model, and to examine the potential mechanism underlying the effect of ephrin-B3 on cell proliferation and the reelin pathway in pilocarpine-induced SE rats.

\section{Materials and methods}

Pilocarpine-induced SE. The animals were provided by the Shanghai Laboratory Animal Center (Shanghai, China) and housed at the Experimental Animal Center of Central South University (Changsha, China) at a temperature of $23 \pm 1^{\circ} \mathrm{C}$ with a regular 12-h light/dark cycle and free access to food and water. All animal experiments were performed in accordance with the official recommendations of the Institutional Animal Care and Use Committee of the Institute of Laboratory Animal Science of Central South University (no. 201403142).

A total of 70 healthy, young male Sprague-Dawley rats (aged 6-8 weeks, 200-250 g) were treated with pilocarpine (25 mg/kg, i.p., Sigma-Aldrich; EMD Millipore, Billerica, MA, USA) to induce epilepsy. The rats were administered with lithium chloride (127 mg/kg, i.p., Sigma-Aldrich; EMD Millipore) $18-22 \mathrm{~h}$ prior to pilocarpine injection. According to Racine's classification, SE was defined as continuous seizures lasting at least $30 \mathrm{~min}$, and rats classified as Racine stage IV-V that fulfilled the SE criterion were used in the present study (15). Following pilocarpine injection, the rats spent $\sim 30$ min in the SE phase in Racine stage IV or V. The matched controls $(n=35)$ were injected i.p. with the same volume of normal saline instead of lithium chloride and pilocarpine, and were used as corresponding controls. All rats were administered with chloral hydrate injection $(3 \mathrm{ml} / \mathrm{kg}$, i.p.; Tonghua Pharmaceutical, Tonghua, China) to terminate behavioral seizures or limit behavioral seizures.

Ephrin-B3 stimulation experiment. On day 7 following the onset of SE, the epileptic and control rats were anesthetized with $10 \%$ chloral hydrate $(3 \mathrm{ml} / \mathrm{kg}$, i.p.) and were then fixed on a stereotaxic apparatus (RWD Life Science, Shenzhen, China). The skin was shaved and sterilized with iodine complex. The scalp was dissected in the middle line and retracted bilaterally to expose the sagittal and coronal sutures. Based on the Rat Brain Stereotaxic Atlas (16), bilateral hippocampal regions were selected as sites for implantation. Two vertical bone holes were drilled symmetrically $1.6 \mathrm{~mm}$ adjacent to the sagittal suture and $3.6 \mathrm{~mm}$ posterior to the anterior fontanel, with the anterior fontanel as the center. A micro-osmotic pump (ALZET ${ }^{\circledR}$; DURECT Corporation, Cupertino, CA, USA) connected to a cannula (ALZET ${ }^{\circledR}$ brain infusion kit) was inserted $2.8 \mathrm{~mm}$ under the dura, and an infusion of Eph B3-Fc suspension (recombinant mouse EphB3-Fc chimera; $50 \mu \mathrm{g} / \mathrm{ml}$; R\&D Systems, Inc., Minneapolis, MN, USA) or Fc $(50 \mu \mathrm{g} / \mathrm{ml}$; Jackson ImmunoResearch Laboratories, Inc., West Grove, PA, USA) were injected into the brain for 7 days. The cannula was cemented onto the skull with dental acrylic. Following removal of the pumps from the skull, the wound was washed repeatedly with sterile saline and was sutured closed. All rats survived, and remained active following surgery.

Behavioral and electroencephalography (EEG) recordings. Spontaneous behavioral seizures were observed in all animals, including controls, following the induction of SE and were usually detected for 60 days between 9 and 11 a.m., and between 3 and 5 p.m. each day using video monitoring. The recordings of the spontaneous seizures consisted of the latency period of SRS, seizure duration, frequency and intensity.

For the EEG recording, the rats were anesthetized using a mixture of $3 \%$ isoflurane in $30 \%$ oxygen and $70 \%$ nitrous oxide, and maintained with $1.5 \%$ isoflurane, and the animal was then fixed into the stereotaxic apparatus (RWD Life Science, Shenzhen, China). Electrodes were placed at the following positions on the skull using an electro-microdriver (RWD Life Science): $2.5 \mathrm{~mm}$ anterior to the bregma, $2.5 \mathrm{~mm}$ bilateral to the midline and $2.5 \mathrm{~mm}$ posterior to the lambda on the midline). The electrodes were then fixed with dental cement. Following recovery for 3 days, EEG monitoring was performed to evaluate the frequency and duration of EEG seizures. An EEG seizure was identified according to the definition of a period of consistent and repetitive changes in amplitude and frequency of electrical activity for at least $10 \mathrm{sec}$, which was different from interictal activity (17). Data were collected and analyzed using an acquisition system (Physical Signal Recorder RM6240; Chengdu Instrument Factory, Chengdu, China).

Histological examination. The rats were deeply anesthetized and then transcardially perfused with a successive 
administration of $0.9 \%$ sterile saline and $4 \%$ paraformaldehyde (PFA) in $0.1 \mathrm{M}$ PBS ( $\mathrm{pH} 7.4 ; 4^{\circ} \mathrm{C}$ ). Following perfusion, the brains were removed from the skull and postfixed in $4 \%$ PFA for $24 \mathrm{~h}$ at $4^{\circ} \mathrm{C}$. Subsequently, the brains were rinsed in PBS and cryoprotected in $30 \%$ sucrose solution at $4^{\circ} \mathrm{C}$ for $72 \mathrm{~h}$. In particular, the coronal slices involving the middle region of the dorsal hippocampus were collected from between -3.6 and $-4.0 \mathrm{~mm}$ to the bregma and sectioned into $25 \mu \mathrm{m}$-thick sections for staining using a freezing microtome (Leica CM 1850; Leica Microsystems GmbH, Wetzlar, Germany).

Immunohistochemical labeling was used to evaluate the protein distribution and expression. Based on standard protocols, the sections were rinsed in PBS, and then incubated in $0.1 \%$ hydrogen peroxide for $30 \mathrm{~min}$ to minimize endogenous peroxidase activity. The sections were then washed three times in PBS, placed in citrate buffer ( $\mathrm{pH}$ 6.0) which had been heated to $90^{\circ} \mathrm{C}$, and then maintained for $20 \mathrm{~min}$ at room temperature to retrieve antigen. Following three washes with PBS, the sections were blocked with $10 \%$ normal goat serum (Sigma-Aldrich, Merck KGaA; Darmstadt, Germany) and avidin (200 $\mu \mathrm{l} / \mathrm{ml}$; Avidin/Biotin blocking kit; Vector Laboratories, Burlingame, CA, USA) in PBS for $2 \mathrm{~h}$ at room temperature. Subsequently, the sections were incubated with primary rabbit ephrin-B3 (1:50, sc-271328; Santa Cruz Biotechnology, Inc., Dallas, Texas, USA); mouse reelin (1:50, sc-25346; Santa Cruz Biotechnology, Inc.); and rabbit p-Dab1 (1:50, Tyr232; Affinity Biologicals, Inc., Ancaster, ON, Canada) antibodies diluted in a solution containing $10 \%$ serum and avidin in PBS at $4^{\circ} \mathrm{C}$ for $48 \mathrm{~h}$. Following rinsing in PBS, the sections were incubated in biotinylated anti-mouse or anti-rabbit or anti-goat secondary antiserum (1:200, 5260-0045, 5260-0038 and 5260-0035; KPL, Inc., Gaithersburg, MA, USA) for $1 \mathrm{~h}$ at room temperature. Following washing in PBS, the sections were incubated with the ABC reagent kit (VECTASTAIN ${ }^{\circledR}$, Vector Laboratories) for $2 \mathrm{~h}$, visualized using DAB (Wuhan Boster Biological Technology, Ltd., Wuhan, China), and counterstained with hematoxylin. Images of the sections were captured using a Leica light microscope (Leica DM5000 B; Leica Microsystems $\mathrm{GmbH}$ ) and analyzed using the ImagePro Plus 6.0 software package (Media Cybernetics, Inc., Rockville, MD, USA) by two investigators, in a blinded-manner, to calculate the mean optical density of immunoreactive products in each microscopic view (magnification, x40).

Immunofluorescent labeling was performed based on standard protocols as described previously (2). The sections were incubated with primary antibodies, including goat doublecortin protein (DCX, 1:125; sc-8066; Santa Cruz Biotechnology, Inc.) and rabbit neuronal nuclei (NeuN, 1:1,000; ab177487; Abcam, Cambridge, MA, USA) at $4^{\circ} \mathrm{C}$ overnight and visualized with appropriate Alexa Fluor-conjugated secondary antibodies (1:1,000; A32723 and A32732; Invitrogen, Thermo Fisher Scientific, Inc., Waltham, MA, USA) at room temperature for 6h. All sections were counterstained with 4',6-diamidino-2-phenylindole (DAPI; Wuhan Boster Biological Technology, Ltd.) and images were captured using a laser scanning confocal microscope (LSM 510; Leica Microsystems GmbH). For quantification analysis, five microscopic fields were selected to observe the distribution of newborn neurons and calculate the cell counts in each view (magnification, x40) using the ImagePro Plus 6.0 software package (Media Cybernetics,
Inc.) by two investigators in a blinded manner. The positive cell counts were normalized to the total number of cells in the regions analyzed in the hippocampus.

SYBR Green-based reverse transcription-quantitative polymerase chain reaction ( $R T-q P C R)$ analysis. The rats were sacrificed 7, 14 and 60 days following the termination of SE, and the hippocampus was rapidly dissected and transferred to liquid nitrogen. All the hippocampal samples were stored at $-80^{\circ} \mathrm{C}$ until processing. Total RNA was extracted using TRIzol reagent (Invitrogen; Thermo Fisher Scientific, Inc.), and reverse transcribed into cDNA using a RevertAid ${ }^{\mathrm{TM}}$ First-Strand complementary DNA (cDNA) synthesis kit (Thermo Fisher Scientific, Inc.). The qPCR sample contained the following: $1 \mu \mathrm{l}$ cDNA, $1 \mu \mathrm{l}$ sense primer, $1 \mu \mathrm{l}$ antisense primer, 9.5 $\mu \mathrm{l}$ RNase-free water and 12.5 $\mu \mathrm{l}$ Maxima ${ }^{\circledR}$ SYBRGreen/ROX qPCR Master mix (Thermo Fisher Scientific, Inc.) to obtain a $25 \mu \mathrm{l}$ reaction volume. The sequences of PCR primers and PCR product lengths were as follows: Ephrin-B3 (NM_001406), forward 5'-ACTCAGCCTGGAGCCTGTCTA C-3' and reverse 5'-CGATCTGAGGGTAAAGCACGTA-3' (212 bp); and $\beta$-actin (NM_007993.3), forward 5'-AATAAG TGGTTACAGGAAG-3' and reverse 5'-GTATTAAGGCGG AAGATT-3' (164 bp). The reactions were performed using the following cycling conditions: Enzyme activation at $95^{\circ} \mathrm{C}$ for $10 \mathrm{~min}$, followed by 40 cycles of denaturation at $95^{\circ} \mathrm{C}$ for $15 \mathrm{sec}$, annealing at $60^{\circ} \mathrm{C}$ for $1 \mathrm{~min}$ and extension at $72^{\circ} \mathrm{C}$ for $20 \mathrm{sec}$. The relative expression levels were calculated as ratios normalized against those of $\beta$-actin and assessed using the ABI StepOnePlus ${ }^{\mathrm{TM}}$ real-time PCR system (Thermo Fisher Scientific, Inc.). The qPCR data were analyzed using the $2^{-\Delta \Delta C q}$ method as described previously (18).

Western blot analysis. The stored hippocampal samples from the rats sacrificed 7, 14 and 60 days following the termination of SE were homogenized in $400 \mu 1$ RIPA lysis buffer (Beyotime Institute of Biotechnology, Shanghai, China) supplemented with the protease inhibitor PMSF (Beijing Solarbio Science $\&$ Technology Co., Ltd., Beijing, China) and the phosphatase inhibitor PhosSTOP (Roche Diagnostics, Basel, Switzerland). The lysates were centrifuged at $14,000 \times \mathrm{g}$ for $20 \mathrm{~min}$ at $4^{\circ} \mathrm{C}$. Bicinchoninic acid assays were used to determine protein concentrations, as described previously (19). Equal quantities of protein $(50 \mu \mathrm{g})$ extracts were separated by electrophoresis on $12 \%$ polyacrylamide gels at $120 \mathrm{~V}$. The proteins were then transferred onto polyvinylidence difluoride membranes in $120 \mathrm{mM}$ glycine, $125 \mathrm{mM}$ Tris, $0.1 \%$ sodium dodecyl sulfate and $20 \%$ methanol at $150 \mathrm{~mA}$ for $1.5 \mathrm{~h}$. The membranes were blocked in $5 \%$ non-fat dry milk in Tris-buffered saline containing $0.1 \%$ Tween-20 (TBST) at $4^{\circ} \mathrm{C}$. The membranes were then incubated with the following primary antibodies: Anti-ephrin-B3 (1:1,000; sc-271328; Santa Cruz Biotechnology, Inc.); anti-p-Dab1 antibodies (1:1,000; Tyr232; Affinity Biologicals); anti-Dab1 (1:2,000; sc-271136; Santa Cruz Biotechnology, Inc.) overnight at $4^{\circ} \mathrm{C}$. Secondary antibodies (1:2,000; 5220-0283, 5220-0460 and 5450-0010; KPL, Inc.) conjugated to horseradish peroxidase were used at a dilution of 1:1,000 in blocking solution at room temperature for $2 \mathrm{~h}$. The membranes were then washed three times with TBST and labeling was visualized using enhanced chemiluminescence 
reagent (Thermo Fisher Scientific, Inc.). The specific bands visible on images were scanned using the Bio-Rad Gel Doc 2000 imaging system (Bio-Rad Laboratories, Inc., Hercules, CA, USA) and measured with Image Lab 3.0 image analysis software (Bio-Rad Laboratories, Inc.). The relative intensities of ephrin-B3 were normalized to the internal reference protein $\beta$-actin (1:1,000; ABP50151; Beyotime Institute of Biotechnology) and the relative intensity of p-Dab1 was normalized to total Dab1.

Statistical analysis. All results are presented as the mean \pm standard deviation. Statistical analyses were performed using GraphPad Prism 6.0 (GraphPad Software, Inc., La Jolla, CA, USA). All continuous variables were tested to confirm that they fit a normal distribution or homoscedasticity according to the results of Levene's test prior to further analysis. Comparisons among groups were analyzed using two-way analysis of variance (ANOVA), followed by multiple Tukey's post hoc test $(\alpha=0.05)$ to assess the difference between any two groups. EEG data between groups was compared using one-way ANOVA, followed by Tukey's post hoc test. $\mathrm{P}<0.05$ was considered to indicate a statistically significant difference.

\section{Results}

Dynamic expression pattern of ephrin-B3 in rats during the development of SE. The results of the RT-qPCR analysis showed a significant downregulation in the gene expression of ephrin-B3 in the hippocampal tissues of epileptic rats (Ep group) during the three phases of $\mathrm{SE}$ development $(\mathrm{P}<0.05$; Fig. 1A). Following pilocarpine-induced SE, the expression of ephrin-B3 was lower in the 7 days group compared with that in the 60 days group, and the lowest expression was observed in the 14 days group $(\mathrm{P}<0.05)$.

Subsequently, immunohistochemistry was used to clarify the distribution of ephrin-B3 in the rat hippocampus; representative images are shown in Fig. 1B. The results of the immunohistochemistry revealed that eprhin-B3 was prominent in granule cells in the dentate gyrus, subgranular zone and hilus in the hippocampus, was sparse in the inner and outer molecular layers, and was mainly expressed in the cell membrane. Additionally, the expression of ephrin-B3 in the hippocampus was decreased in the 14 days group, compared with that in the 7 days group, but was increased in the 60 days group (Fig. 1C). These results were confirmed by detecting the protein expression of ephrin-B3 by western blot analysis, which showed similar dynamic changes to gene expression (Fig. 1D and E).

Hippocampal neurogenesis in rats during the development of $S E$. To examine hippocampal neurogenesis following pilocarpine-induced SE, DCX and NeuN were used for double-labeling to measure the changes in newborn neurons in the hippocampus post-SE, as they can recognize late mitotic neuronal precursors and newly-generated postmitotic neurons. DCX is a marker of transit-amplifying cells and immature neurons, which begin to have neuronal potential, whereas NeuN labels postmitotic neurons, which have been found to be mature granule cells. The DCX/NeuN-positive cells showed the features of newly generated neurons in the rats; representative images are shown in Fig. $2 \mathrm{~A}$. The number of newborn neurons was significantly increased in the rats following pilocarpine-induced $\mathrm{SE}(\mathrm{P}<0.05$; Fig. $2 \mathrm{~B})$. Consistent with the increased number of newborn neurons, the DCX-positive cells in the Ep rats clustered along the subgranular zone and revealed premature branching of the dendritic outgrowth within the granule cell layer, random and nonradial orientation of the outgrowth, and numerous abnormal neuronal processes when compared with the control group. The images indicated the abnormal positioning of DCX-positive cells in the hippocampus and abnormal morphology of dendritic outgrowth following pilocarpine induction.

Reelin pathway expression in rats during the development of $S E$. As shown in Fig. 3A, the immunohistochemistry results revealed a significant downregulation in the expression of reelin in the rat hippocampus during the three phases of SE, with a lower expression at 7 days, compared with that at 60 days, and the lowest expression observed at 14 days $(\mathrm{P}<0.05$; Fig. 3B). The immunohistochemistry also revealed the distribution of reelin, which was mainly expressed in the interneurons in the dentate gyrus, hilus and subgranular zone, and labeled as numerous punctate structures on the cell membrane. The phosphorylation of Dab1 is necessary for the activation of reelin signaling. The immunohistochemistry revealed that, compared with the control rats, the Ep rats showed significantly decreased expression of $\mathrm{p}$-Dab1 on days 7, 14 and 60 post-SE induction $(\mathrm{P}<0.05$; Fig. 3C and D). The immunohistochemistry also revealed that p-Dabl was labeled in the dentate granule cell body in the hilus and dentate gyrus, the inner molecular layer and stratum radiatum of the hippocampus. In addition, the expression levels of p-Dab1 and total Dab1 were assessed using western blot analysis following pilocarpine-induced SE (Fig. 3E and F). The expression of $\mathrm{p}$-Dab1 was downregulated, whereas that of total Dab1 remained unchanged.

Effects of ephrin-B3 stimulator on hippocampal neurogenesis. To further examine whether ephrin-B3 can affect hippocampal neurogenesis in the hippocampus in Ep rats, a stimulator of ephrin-B3 with EphB3-Fc/Fc-control was injected into the Ep rats 7 days following pilocarpine-induced SE (Fig. 4A). The expression level of ephrin-B3 was confirmed to be upregulated following injection with EphB3-Fc, whereas no changes were found in the Fc-control groups (Fig. 4B). As shown in Fig. 4C and D, following EpB3-Fc injection, the number of newborn neurons was significantly decreased, compared with that following Fc-control treatment $(\mathrm{P}<0.05)$. DCX-labeled cells in the Ep rats post-EphB3-Fc treatment were scattered in the subgranular zone, with short dendrites that extended through $<1 / 3$ of the granular cell layer, compared with those in the Fc-control group. No significant difference was observed in the control groups between the EphB3-Fc and Fc-control treatments. These results suggested that EphB3-Fc reduced the number of newborn neurons and abnormal dendritic outgrowth following pilocarpine-induced SE.

Effects of ephrin-B3 stimulator on the reelin pathway. To further examine whether ephrin-B3 can affect the protein expression levels of reelin and p-Dab1 in the hippocampus of Ep rats, the present study detected the expression levels of reelin 
A

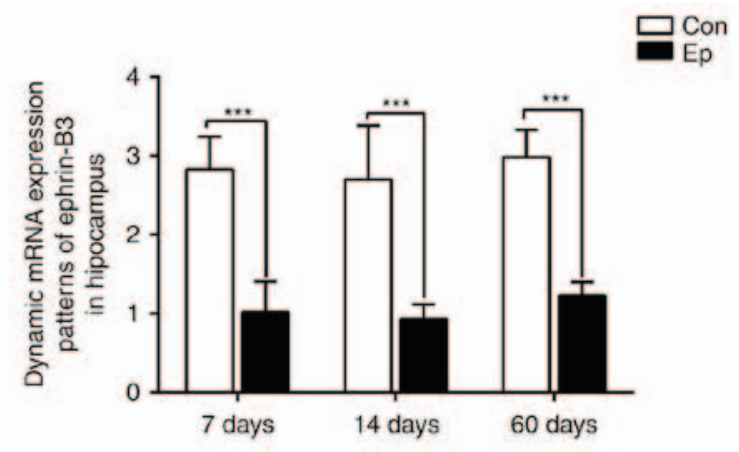

B

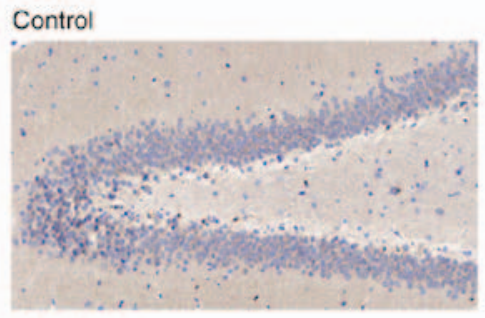

Ep 7 days

\section{Ep 14 days}

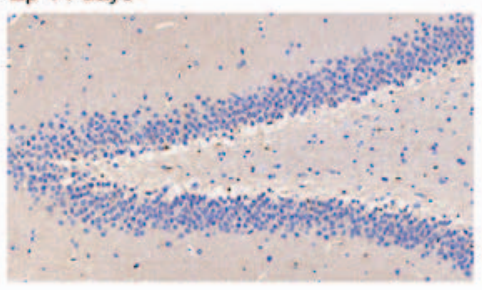

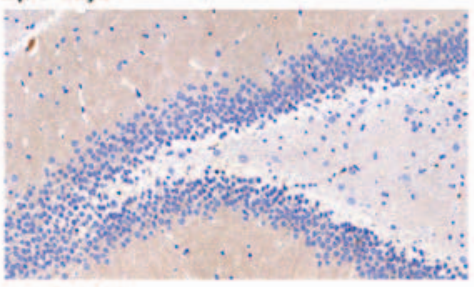

Ep 60 days

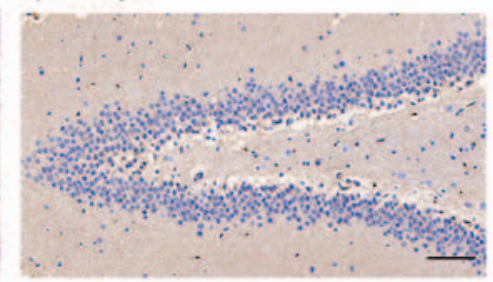

C
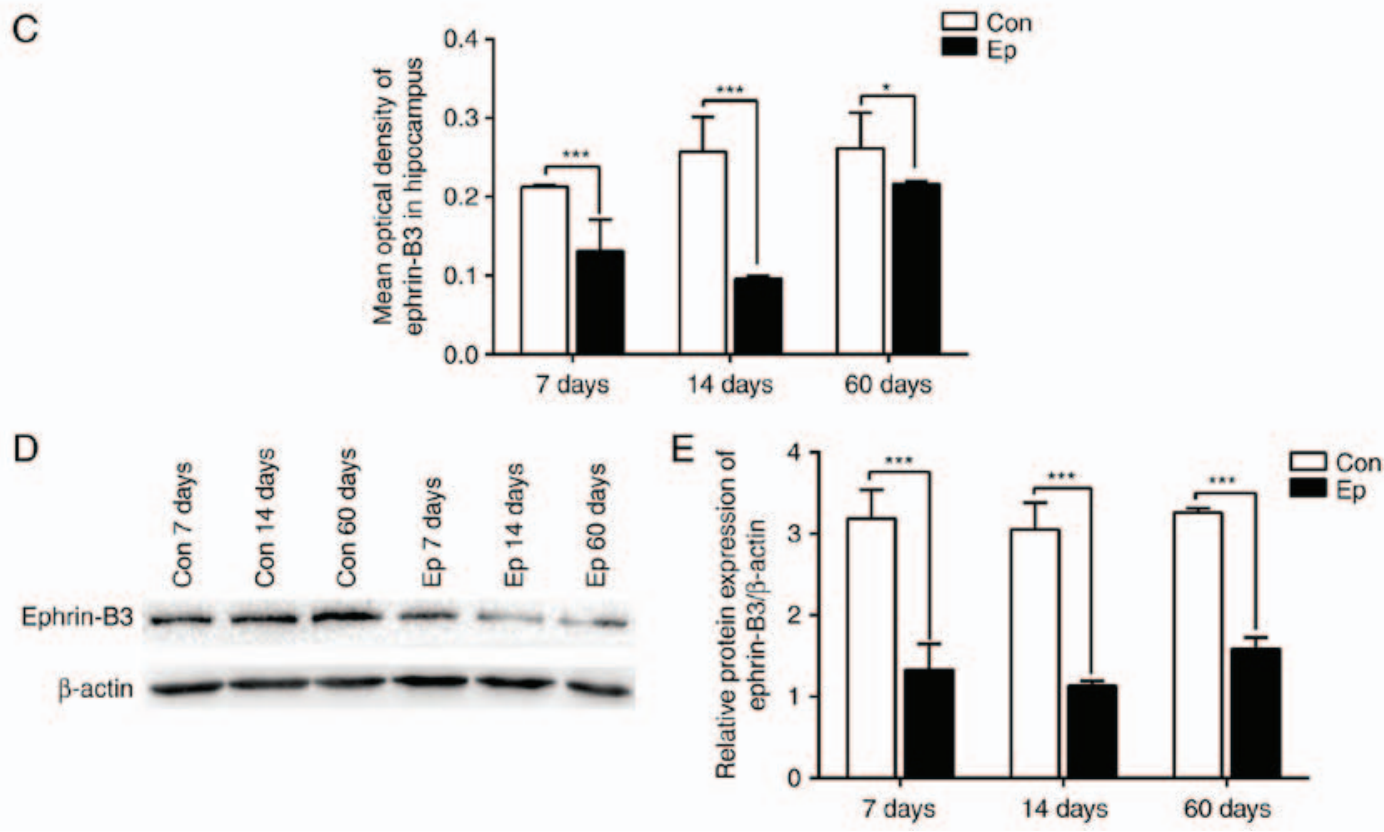

Figure 1. Dynamic expression patterns of ephrin-B3 in the hippocampus of rats during the development of SE. (A) mRNA expression of ephrin-B3 in each group, as assessed by reverse transcription-quantitative polymerase chain reaction analysis. The results showed significant downregulation in the gene expression of ephrin-B3 in the rat hippocampal tissues during the three phases of SE development. (B) Representative immunohistochemical staining of protein expression and distribution of ephrin-B3 in the hippocampus. (C) Results of the mean optical density of ephrin-B3 in the hippocampus. (D) Western blot analysis for the expression of ephrin-B3. (E) Quantitative results of protein expression of ephrin-B3 detected by western blot analysis indicated that Ep reduced the protein expression of Ephrin-B3. Data are presented as the mean \pm standard deviation. Scale bar $=100 \mu \mathrm{m} .{ }^{* * *} \mathrm{P}<0.0001$, ${ }^{*} \mathrm{P}<0.01$ by two-way analysis of variance with Tukey's post hoc test (n=6 rats/per group); Ep, epilepsy; Con, control.

and p-Dab1 following EphB3-Fc injection (Fig. 5). The immunohistochemistry results showed that the injection of ephrin-B3 stimulator markedly increased the expression levels of reelin in the hippocampus of Ep rats post-SE $(\mathrm{P}<0.05)$, whereas no significant increase in the protein expression of reelin was observed in the rats injected with Fc-control (Fig. 5A). In addition, as shown in Fig. 5B and $\mathrm{C}$, the expression of p-Dab1 detected by immunohistochemistry was increased 
A Control

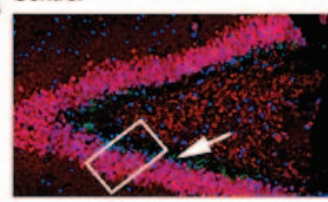

Ep 14 days

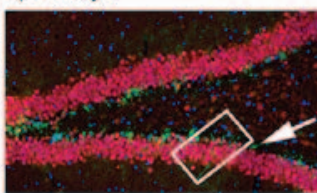

Ep 7 days
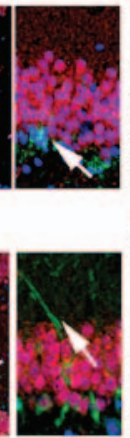

Ep 60 days
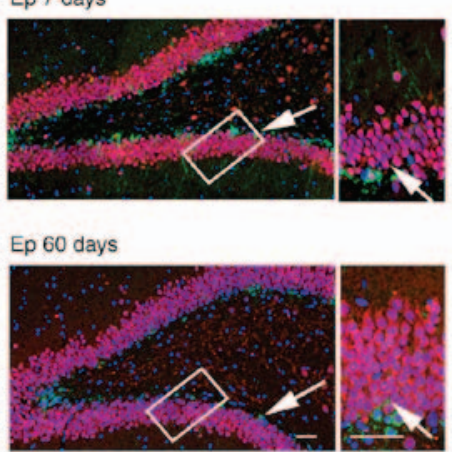

B

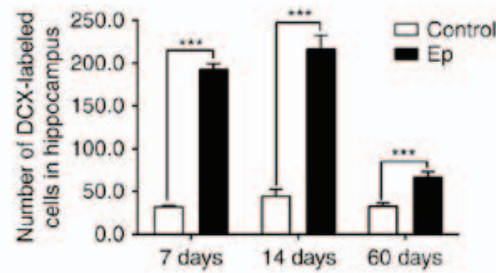

Figure 2. Hippocampal neurogenesis in rats during the development of SE. (A) Representative immunofluorescent results of hippocampal neurogenesis using DCX (green), NeuN (red) and DAPI (blue) antibodies to identify progenitor cells (green) and mature neurons (red). The arrows indicate the DCX/DAPI co-labeled newborn neurons. High-magnification bright-field images are shown in the boxed section. (B) Quantification results of DCX-positive cells in the hippocampus. Ep rats showed more mislocalized progenitors in the hippocampus, compared with the control rats. Data are presented as the mean \pm standard deviation. Scale bar $=50 \mu \mathrm{m} .{ }^{* * *} \mathrm{P}<0.0001$ by two-way analysis of variance with Tukey's post hoc test ( $\mathrm{n}=5$ slices/per group). Ep, epilepsy; DCX, doublecortin; DAPI, 4',6-diamidino-2-phenylindole.
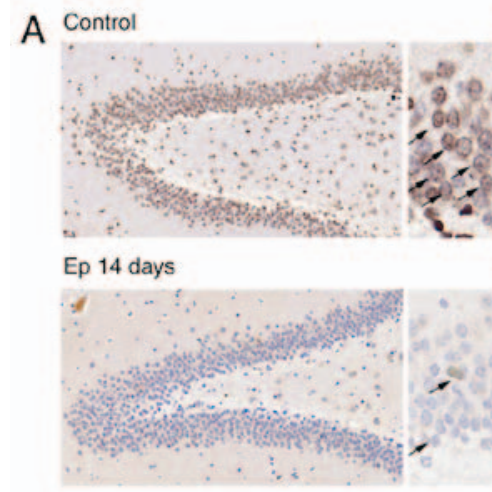

C

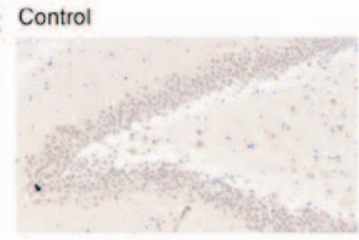

Ep 14 days

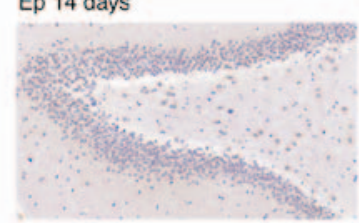

Ep 7 days

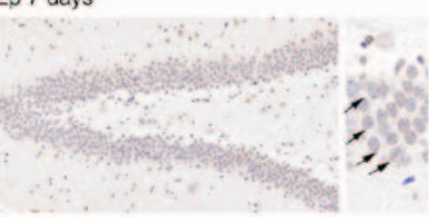

Ep 60 days

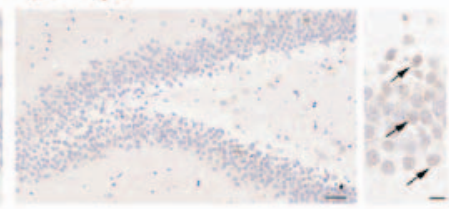

Ep 7 days

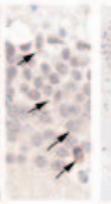

Ep 60 days

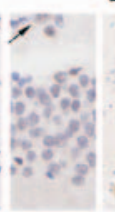

B

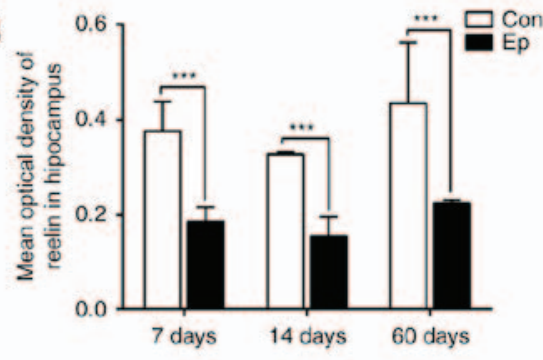

D

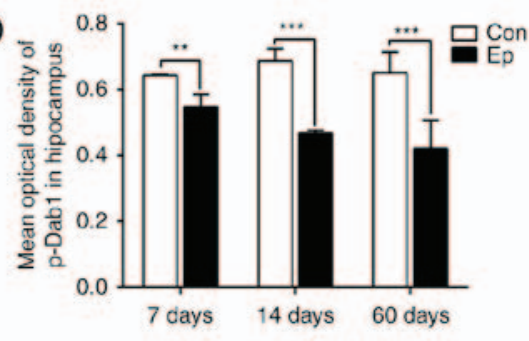

$F$

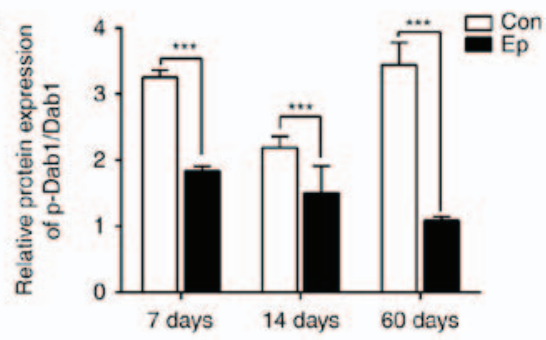

Figure 3. Reelin pathway expression in the hippocampus of rats during the development of SE. (A) Representative immunohistochemical staining of reelin protein in hippocampal tissues during the three phases of SE development. Left: representative immunohistochemical staining of reelin protein (Scale bar=50 $\mu \mathrm{m}$ ); right: High-magnification bright-field images and arrows indicating the immunopositive cells (Scale bar=10 $\mu \mathrm{m}$ ). (B) Bar graph results of reelin protein in hippocampal tissues during the three phases of SE development. (C) Representative immunohistochemical staining of p-Dab1 protein in the hippocampus. Left: representative immunohistochemical staining of p-Dab1 protein (Scale bar=50 $\mu \mathrm{m}$ ); right: high-magnification bright-field images and the arrows indicate the immunopositive cells (Scale bar=10 $\mu \mathrm{m}$ ). (D) Bar graph results of p-Dab1 protein in the hippocampus. (E) Western blot analysis for p-Dab1. $\beta$-actin was used as an endogenous control. (F) Quantification of western blot results of p-Dab1, presented as the p-Dab1/Dab1 ratio. Data are presented as the mean \pm standard deviation. ${ }^{* * *} \mathrm{P}<0.0001,{ }^{* *} \mathrm{P}<0.001$ by two-way analysis of variance with Tukey's post hoc test (n=3 rats/per group). Ep, epilepsy; Con, control; Dab1, disabled 1; p-Dab, phosphorylated Dab1. 
A

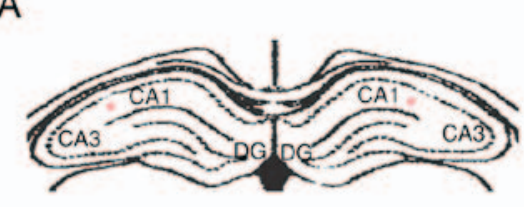

B

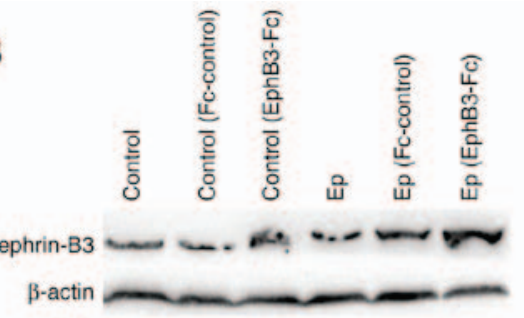

C Control (Fc-control)

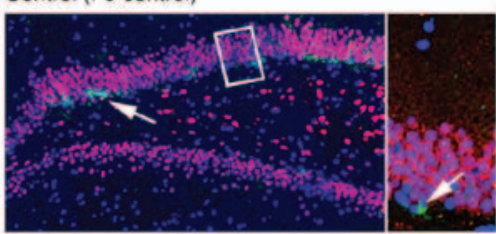

Ep (FC-control)

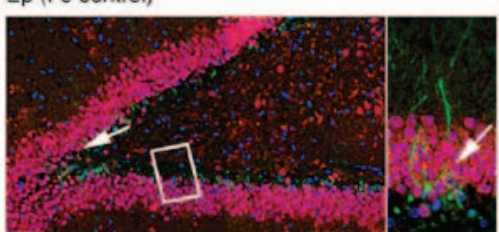

Control (EphB3-Fc)

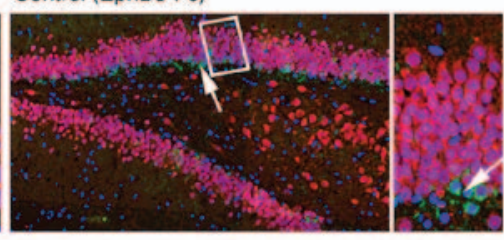

Ep (EphB3-Fc)

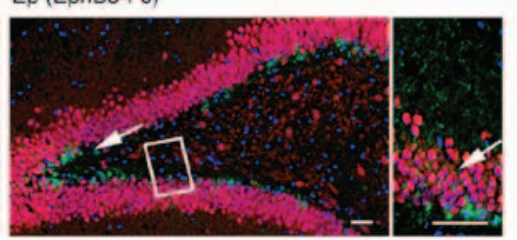

D

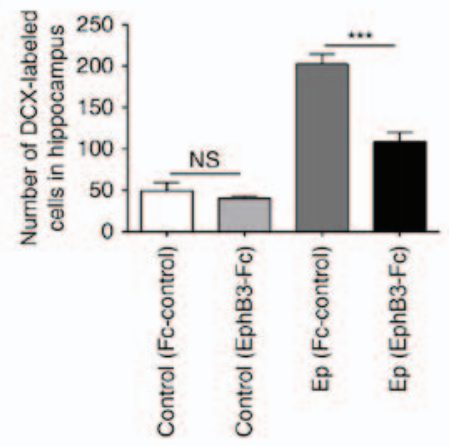

Figure 4. Effect of EphB3-Fc on hippocampal neurogenesis post-SE. (A) Schematic of a typical coronal hippocampal section, illustrating the region of EphB3-Fc injection. Asterisks represent the injection sites. (B) Expression of ephrin-B3 increased following EphB3-Fc infusion, detected by western blot analysis. (C) Representative immunofluorescent results of hippocampal neurogenesis using DCX (green), NeuN (red) and DAPI (blue) antibodies to identify progenitor cells (green) and mature neurons (red). The arrows indicate the DCX/DAPI co-labeled newborn neurons. High-magnification bright-field images are shown in the boxed section. (D) Bar graph results of the immunofluorescent results. Following EphB3-Fc treatment, Ep rats showed fewer DCX-labeled cells, compared with the Fc-control rats, whereas no significant difference was found between EphB3-Fc and Fc-control treatment in the control rats. Data are presented as the mean \pm standard deviation. Scale bar $=50 \mu \mathrm{m} .{ }^{* * *} \mathrm{P}<0.0001, \mathrm{~ns},(\mathrm{P}>0.05)$ by two-way analysis of variance with Tukey's post hoc test ( $\mathrm{n}=5$ slices/per group). Ep, epilepsy; DCX, doublecortin; DAPI, 4',6-diamidino-2-phenylindole; ns, not significant.

following EphB3-Fc treatment $(\mathrm{P}<0.05)$, whereas Fc-control treatment had no effect on the level of p-Dab1 post-SE. The expression of p-Dab1 was also confirmed using western blot analysis (Fig. 5D and E), which also showed upregulation in the hippocampus of Ep rats following EphB3-Fc treatment. By contrast, the hippocampal level of total Dab1 was not altered following EphB3-Fc or Fc-control treatment. No significant difference was found in the expression of reelin or p-Dab1 in the control rats between the EphB3-Fc and Fc-control treatment groups.

Characteristics of behaviors and EEG following ephrin-B3 stimulation treatment in the rat model. All the rats were monitored to record their behaviors and EEG. The control animals without injections of pilocarpine did not exhibit seizure activities. Following several minutes of pilocarpine injection, the rats exhibited facial muscle, forelimb and tail clonus, and ataxic lurching, head bobbing and wet dog shakes. In the latent stage of SE (14 days post-SE), no behavioral changes or seizures were observed in the Ep rats. Spontaneous recurrent seizures occurred at 60 days post-SE in the Ep rats (chronic stage of SE). However, following EphB3-Fc treatment, it was found that the Ep rats exhibited reduced average latency periods to the onset of seizure, seizure thresholds and seizure frequencies, compared with the Fc-control-treated rats $(\mathrm{P}<0.05)$; whereas $\mathrm{Fc}$-control treatment had no effect on seizures. No significant difference was found in seizure activities of the control rats following EphB3-Fc or Fc-control treatment (data not shown).
EEG recordings that showed high frequency and amplitude, poly-spike paroxysmal electrical activities, and persisted for at least $10 \mathrm{sec}$ were considered to be EEG seizures (Fig. 6A-C). No EEG changes or seizures were observed in the control rats. The pilocarpine-induced rats exhibited EEG seizures characterized by a recruiting rhythmic activity followed by spiking activity of progressively increased frequency and voltage. Treatment with EphB3-Fc significantly suppressed the frequency, duration and the firing amplitudes of spontaneous seizures in the Ep rats. However, no significant differences were found in frequency, duration or epileptiform activity in the control rats following EphB3-Fc or Fc-control treatment.

\section{Discussion}

The present study is the first, to the best of our knowledge, to demonstrate the role of ephrin-B3 in neurogenesis and the reelin pathway during the development of SE in epileptic rats. By integrating the data, it was found that the expression of ephrin-B3 was significantly downregulated in epileptic rats; the expression of ephrin-B3 was lower in the acute phase, compared with that in the chronic phase, and was lowest in the latent phase. The reelin pathway showed similar dynamic changes to the expression of ephrin-B3. The pilocarpine-induced rats exhibited increased hippocampal neurogenesis during the spontaneous period of SE. The effects of ephrin-B3 on hippocampal neurogenesis and reelin pathway expression levels were examined, and it was found that, following the administration of exogenous ephrin-B3, the 
A Control (Fc-control)
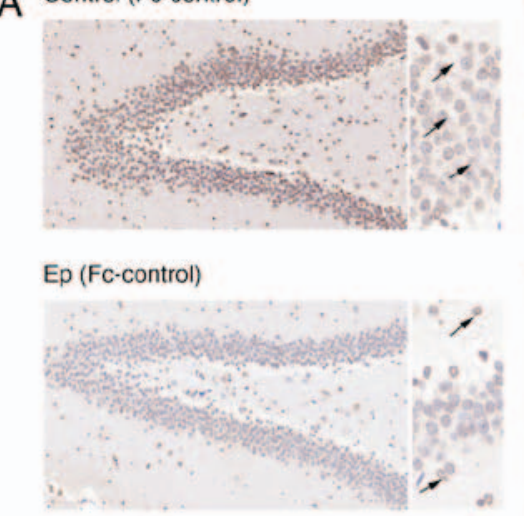

C
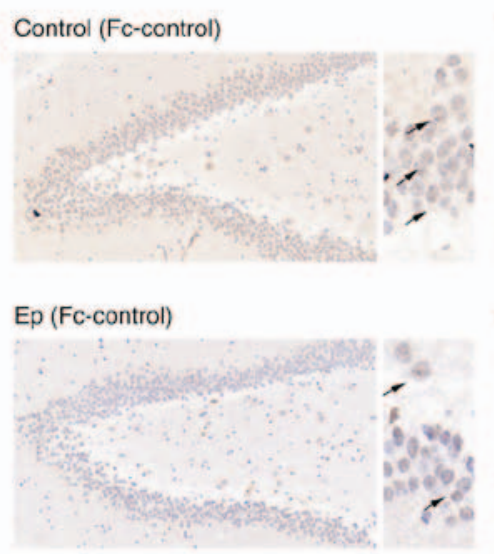

Control (EphB3-Fc)

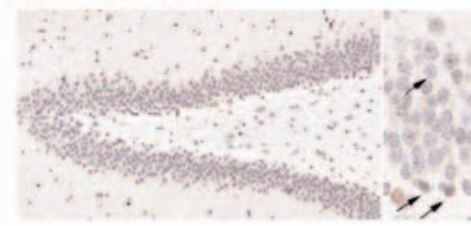

Ep (EphB3-Fc)

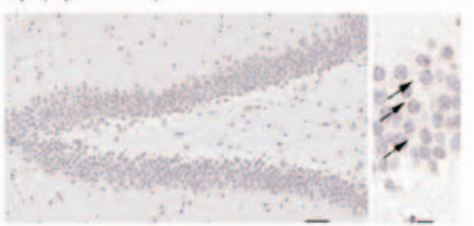

Control (EphB3-Fc)

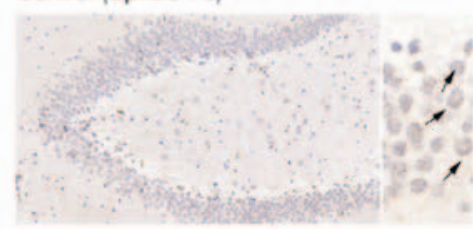

Ep (EphB3-Fc)

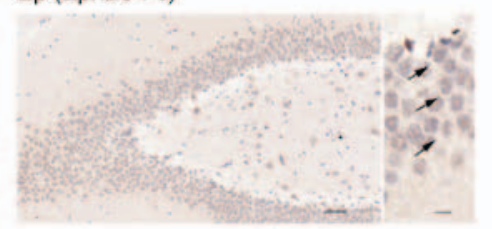

B

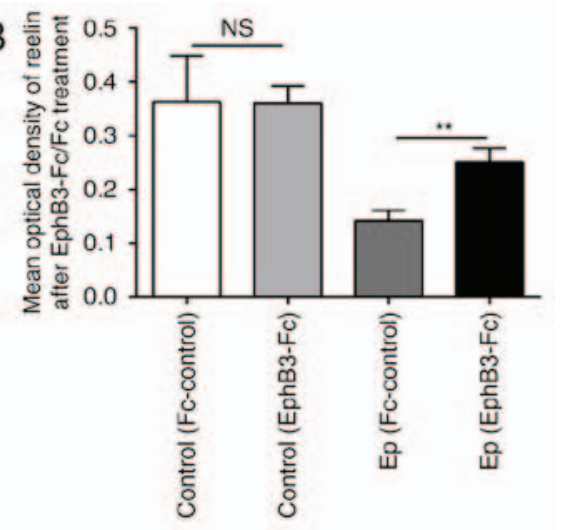

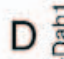
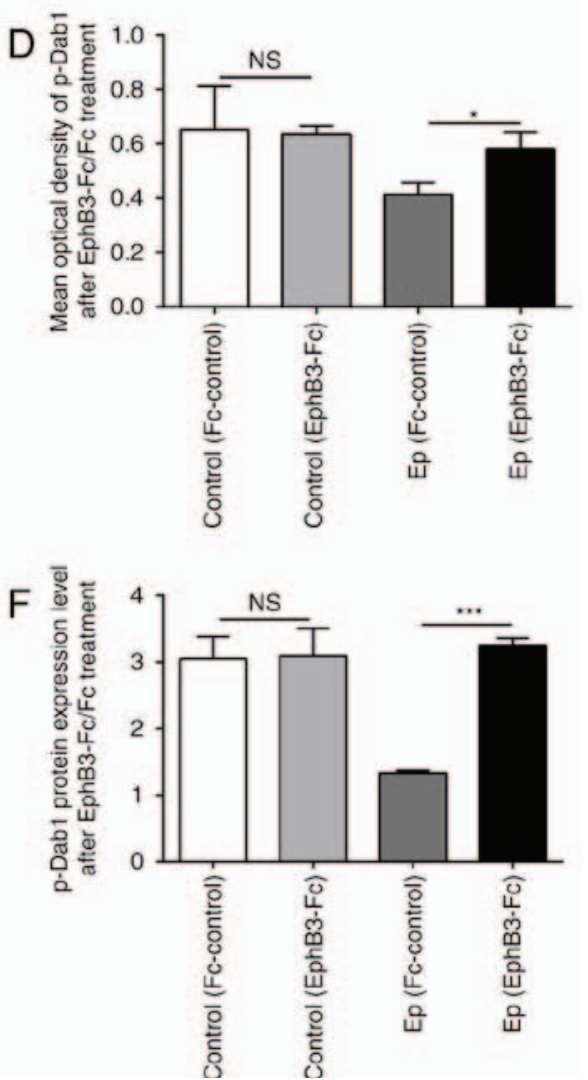

Figure 5. Effect of EphB3-Fc on reelin pathway expression in the hippocampus post-SE. (A) Representative immunohistochemical staining of reelin following EphB3-Fc/Fc-control treatment. Left: representative immunohistochemical staining of reelin (scale bar=50 $\mu \mathrm{m}$ ); right: high-magnification bright-field images and the arrows indicate the immunopositive cells (scale bar=10 $\mu \mathrm{m}$ ). (B) Bar graph results of the immunohistochemistry assay of reelin protein. (C) Representative immunohistochemical staining of p-Dab1 following EphB3-Fc/Fc-control treatment. Left: representative immunohistochemical staining of p-Dab1 (scale bar=50 $\mu \mathrm{m}$ ); right: high-magnification bright-field images and the arrows indicate the immunopositive cells (scale bar=10 $\mu \mathrm{m}$ ). (D) Bar graph results of the immunohistochemistry assay of p-Dab1. (E) Western blot analysis for expression of p-Dab1. $\beta$-actin was used as an endogenous control. (F) Quantification of p-Dab1 expression by western blot analysis. Data are presented as the mean \pm standard deviation. ${ }^{* * * *} \mathrm{P}<0.0001,{ }^{* * *} \mathrm{P}<0.001,{ }^{*} \mathrm{P}<0.01$, $\mathrm{ns}=\mathrm{P}>0.05$ by two-way analysis of variance with Tukey's post hoc test ( $\mathrm{n}=3$ rats/per group). Ep, epilepsy; Con, control; Dab1, disabled 1; $\mathrm{p}$-Dab, phosphorylated Dab1; ns, not significant.

epileptic rats showed subsequent increases in hippocampal reelin pathway expression and reduced neurogenesis, accompanied by the suppression of spontaneous seizures. These results suggested the underlying functions of ephrin-B3 on the modulation of the reelin pathway, and the suppression of neurogenesis and seizure activity in epilepsy.

Increasing investigations have started to investigate the role of Eph receptor and its ligand ephrins in the central nervous system (20). Ephrin-B3, the ligand of EphB receptor is critical in the regulation of cell signaling, neuronal migration and cell proliferation, dendritic spine maturity, formation of excitatory synapses, and synaptic plasticity in the central nervous system $(21,22)$. In a previous animal study, mice lacking ephrin-B3 or EphB1 receptor exhibited disrupted hippocampal neurogenesis, including polarity, cell positioning and proliferation (8). In the present study, it was demonstrated that ephrin-B3 was significantly decreased post-SE in epileptic rats, which corroborated with the previous finding that the involvement of ephrin-B3 is an important regulator of neuronal cell positioning in the central nervous system (12). The present study also found that the expression of ephrin-B3 was marginally increased in epileptic rats in the chronic phase. A possible 

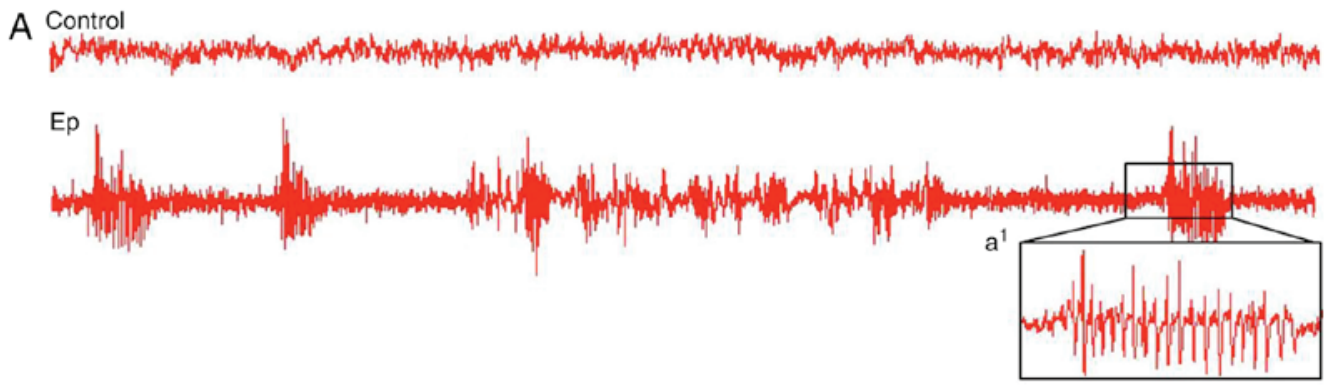

B Ep (Fc-control)
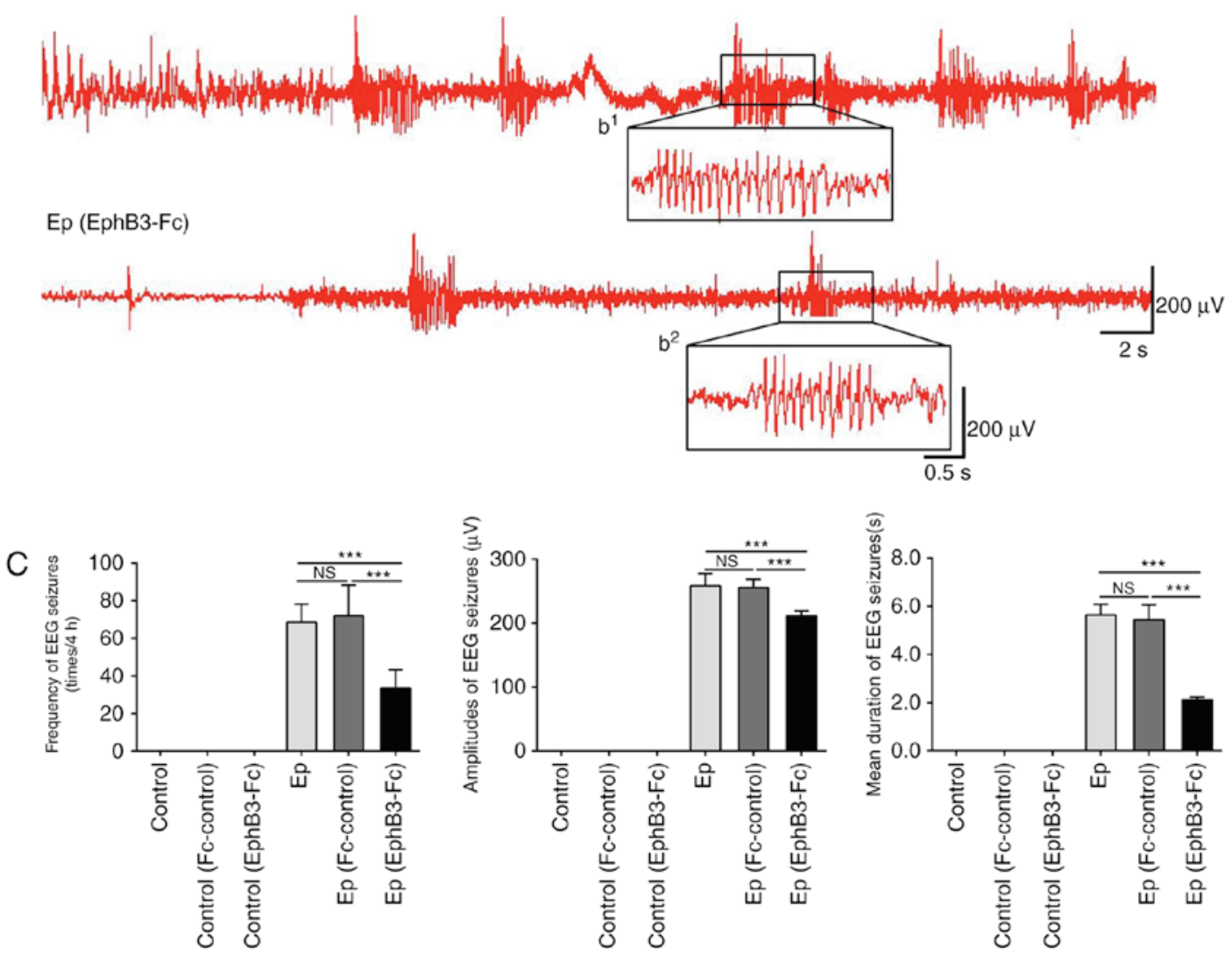

Figure 6. EEG recordings. (A) EEG activity of control rats and epileptic rats. Ep rats showed typical EEG seizures ( $\mathrm{a}^{1}$ ). (B) Spontaneous EEG seizures in Ep rats following EphB3-Fc/Fc-control treatment. Compared with the Fc-control treated rats, treatment with EphB3-Fc significantly suppressed the frequency of spontaneous seizures, as demonstrated by reduced epileptiform activity in the Ep rats $\left(\mathrm{b}^{1} \mathrm{vs} . \mathrm{b}^{2}\right)$. Each box indicated the enlargement of the corresponding EEG sample. (C) Quantification of the frequency of EEG seizures, seizure amplitude and mean duration of EEG seizures. Data are presented as the mean \pm standard deviation. ${ }^{* * * *} \mathrm{P}<0.0001, \mathrm{~ns}=\mathrm{P}>0.05$ by two-way analysis of variance with Tukey's post hoc test ( $\mathrm{n}=3$ rats/per group). Ep, epilepsy; EEG, electroencephalography.

explanation for this increase is that ephrin-B3 binding to its cognate receptor can induce a small depression of excitatory synaptic transmission in cultured hippocampal neurons (23). The increase of ephrin-B3 may serve as a compensatory mechanism to maintain homeostatic balance in the chronic stage of SE.

Hippocampal neurogenesis in several animal models of epilepsy can result in alteration of the excitability of neurons, which contributes to spontaneous recurrent seizures $(24,25)$. Seizures can activate progenitor cells in the subgranular zone of the dentate gyrus in the hippocampus, and these newborn neurons integrate into the hippocampal circuitry and contribute to hippocampal network plasticity, thereby affecting epilepsy $(25,26)$. Consistent with the findings in the present study, Song et al also found that newborn neurons exhibited abnormal dendritic development, including longer apical and basal dendrites, and were accompanied by long-term seizure activity in an intraventricular kainic acid model of epilepsy (27). Of note, the hippocampal neurogenesis and neuronal migration stimulated by SE were prominent at least within 28 days, which coincided with the finding in the latent phase in the present study (24). By comparing the number and features of newborn neurons following EphB3-Fc injection, the epileptic rats showed a reduction in proliferation, altered migration and relatively normal branching of newborn neurons. These results further support the hypothesis that ephrin-B3 is involved in regulating the proliferation and migration of neural progenitors during the development of SE.

Furthermore, the present study examined the role of ephrin-B3 in the regulation of reelin pathway during the 
development of SE. In association with other observations in human temporal lobe epilepsy specimens (28), the present study showed the downregulation of reelin pathway expression in epileptic rats, which indicated that this pathway may also be involved in neuronal migration post-SE. Several studies have suggested that reelin is also associated with epilepsy; for example, reelin deficiency has an effect on granule cell dispersion in mesial temporal lobe epilepsy (29), and heterozygous reelin mutation is associated with a clinical phenotype of temporal lobe epilepsy in humans (30). Baek et al also found that the misexpression of reelin can lead to a migration defect in neurons of focal malformations in cortical development in the human and mouse brain (31). In addition, a previous study demonstrated that Dab1-deficient mice exhibit interictal epileptiform abnormalities and a reduced latency to pilocarpine-induced SE (32), which is similar to the findings in the present study. However, following the administration of exogenous ephrin-B3 with EphB3-Fc in the present study, subsequent upregulation in the expression of reelin and downstream p-Dab1 were observed in the hippocampus. This finding is consistent with a report by Senturk et al, which showed the ephrin-B3 can activate the reelin pathway in reelin-knockout mice (12). However, the findings in the present study are the first, to the best of our knowledge, to demonstrate the dynamic changes in the reelin pathway and the role of ephrin-B3 in modulating the reelin pathway during the development of SE.

There were limitations to the present study. First, only one time point was examined, rather than longitudinal behavioral and molecular changes following injection. This is due to our findings that ephrin-B3 and reelin decreased from 7 days and were expressed at its lowest level in 14 days. Secondly, the present study only showed the expression of the reelin pathway following exogenous ephrin-B3 injection, with no intervening of the reelin pathway; future investigations aim to include interventional methods to further investigate the downstream proteins and confirm the effect of ephrin-B3 on the reelin pathway. Finally, preclustered Eph-B3-Fc molecules increase ephrin-B3 protein by its bidirectional signaling (33), therefore, it is possible to cluster ephrin-B1 or B2 (7). However, ephrin-B3 is the primarily activated protein by EphB3-Fc in cortical and hippocampal neurons according to previous studies (12). Therefore, may be useful to examine the function of ephrin-B1 or B2 and attempt other genetic methods, including ephrin-B3 transgenic mice, in the future.

In conclusion, the observations in the present study provide evidence that ephrin-B3 is key in the amelioration of neurogenesis and suppression of neuronal excitation in epilepsy. Furthermore, ephrin-B3 was found to be involved in modulation of the reelin signaling pathway during the development of epilepsy. These findings support the hypothesis that the administration of ephrin-B3 may assist in preventing recurrent seizures, at least in an animal model. Further investigations are required to validate whether, and to what extent, the results obtained in the present study can be extrapolated to patients.

\section{Acknowledgements}

The authors would like to thank Miss Qiuxiang $\mathrm{Li}$ at the Department of Neurology, Xiangya Hospital, Central South
University (Changsha, China) for tissue preparation and also Dr Zhaohui Luo and Dr Zhiguo Wu from the Department of Neurology, Xiangya Hospital, Central South University (Changsha, China) for their outstanding technical assistance with experimental apparatus.

\section{Funding}

This study was supported by grants from National Natural Science Foundation of China (grant nos. 81771407, 81100967 and 81371435).

\section{Availability of data and materials}

The datasets used and analyzed during the current study are available from the corresponding author on reasonable request.

\section{Authors' contributions}

BX and LF designed the research. TTL performed the majority of the experiments. YL and YS contributed to the study design and analyzed the data. TTL, BX and LF wrote the article. All authors read and approved the final manuscript.

\section{Ethics approval and consent to participate}

All experiments performed in the present study involving animals were performed with the ethical approval of the Institutional Animal Care and Use Committee of the Institute of Laboratory Animal Science of Central South University (no. 201403142).

\section{Consent for publication}

Not applicable.

\section{Competing interests}

The authors declare that they have no competing interests.

\section{References}

1. Moshe SL, Perucca E, Ryvlin P and Tomson T: Epilepsy: New advances. Lancet 385: 884-898, 2015.

2. Liu TT, Feng L, Liu HF, Shu Y and Xiao B: Altered axon initial segment in hippocampal newborn neurons, associated with recurrence of temporal lobe epilepsy in rats. Mol Med Rep 16: 3169-3178, 2017.

3. Li Y, Peng Z, Xiao B and Houser CR: Activation of ERK by spontaneous seizures in neural progenitors of the dentate gyrus in a mouse model of epilepsy. Exp Neurol 224: 133-145, 2010.

4. von Bohlen und Halbach O: Immunohistological markers for proliferative events, gliogenesis, and neurogenesis within the adult hippocampus. Cell Tissue Res 345: 1-19, 2011.

5. Parent JM and Kron MM: Neurogenesis and Epilepsy. 2012.

6. Ishii K, Kubo K, Endo T, Yoshida K, Benner S, Ito Y, Aizawa H, Aramaki M, Yamanaka A, Tanaka K, et al: Neuronal Heterotopias affect the activities of distant brain areas and lead to behavioral deficits. J Neurosci 35: 12432-12445, 2015.

7. Klein R and Kania A: Ephrin signalling in the developing nervous system. Curr Opin Neurobiol 27C: 16-24, 2014.

8. Chumley MJ, Catchpole T, Silvany RE, Kernie SG and Henkemeyer M: EphB receptors regulate stem/progenitor cell proliferation, migration, and polarity during hippocampal neurogenesis. J Neurosci 27: 13481-13490, 2007. 
9. Furne C, Ricard J, Cabrera JR, Pays L, Bethea JR, Mehlen P and Liebl DJ: EphrinB3 is an anti-apoptotic ligand that inhibits the dependence receptor functions of EphA4 receptors during adult neurogenesis. Biochim Biophys Acta 1793: 231-238, 2009.

10. Antion MD, Christie LA, Bond AM, Dalva MB and Contractor A: Ephrin-B3 regulates glutamate receptor signaling at hippocampal synapses. Mol Cell Neurosci 45: 378-388, 2010.

11. Forster E: Reelin, neuronal polarity and process orientation of cortical neurons. Neuroscience 269: 102-111, 2014.

12. Senturk A, Pfennig S, Weiss A, Burk K and Acker-Palmer A: Ephrin Bs are essential components of the Reelin pathway to regulate neuronal migration. Nature 472: 356-360, 2011.

13. Leeb C, Eresheim C and Nimpf J: Clusterin is a ligand for apolipoprotein $\mathrm{E}$ receptor 2 (ApoER2) and very low density lipoprotein receptor (VLDLR) and signals via the Reelin-signaling pathway. J Biol Chem 289: 4161-4172, 2014.

14. Bosch C, Masachs N, Exposito-Alonso D, Martínez A, Teixeira CM, Fernaud I, Pujadas L, Ulloa F, Comella JX, DeFelipe $\mathrm{J}$, et al: Reelin regulates the maturation of dendritic spines, synaptogenesis and glial ensheathment of newborn granule cells. Cereb Cortex 26: 4282-4298, 2016.

15. Racine RJ: Modification of seizure activity by electrical stimulation. II. Motor seizure. Electroencephalogr Clin Neurophysiol 32: 281-294, 1972.

16. Khazipov R, Zaynutdinova D, Ogievetsky E, Valeeva G, Mitrukhina O, Manent JB and Represa A: Atlas of the postnatal rat brain in stereotaxic coordinates. Front Neuroanat 9: 161, 2015

17. Kanamori K: Faster flux of neurotransmitter glutamate during seizure-Evidence from 13C-enrichment of extracellular glutamate in kainate rat model. PLoS One 12: e0174845, 2017.

18. Livak KJ and Schmittgen TD: Analysis of relative gene expression data using real-time quantitative PCR and the $2^{-\Delta \Delta C_{\mathrm{T}}}$ method. Methods 25: 402-408, 2001.

19. Wu Q, Li Y, Shu Y, Feng L, Zhou L, Yue ZW, Luo ZH, Wu ZG and Xiao B: NDEL1 was decreased in the CA3 region but increased in the hippocampal blood vessel network during the spontaneous seizure period after pilocarpine-induced status epilepticus. Neuroscience 268: 276-283, 2014.

20. Lisabeth EM, Falivelli G and Pasquale EB: Eph receptor signaling and ephrins. Cold Spring Harb Perspect Biol 5, 2013.

21. Hruska M and Dalva MB: Ephrin regulation of synapse formation, function and plasticity. Mol Cell Neurosci 50: 35-44, 2012.

22. Ricard J, Salinas J, Garcia L and Liebl DJ: EphrinB3 regulates cell proliferation and survival in adult neurogenesis. Mol Cell Neurosci 31: 713-722, 2006.

23. Piccinin S, Cinque C, Calo L, Molinaro G, Battaglia G, Maggi L, Nicoletti F, Melchiorri D, Eusebi F, Massey PV, et al: Interaction between Ephrins and mGlu5 metabotropic glutamate receptors in the induction of long-term synaptic depression in the hippocampus. J Neurosci 30: 2835-2843, 2010.
24. Shu Y, Xiao B, Wu Q, Liu T, Du Y, Tang H, Chen S, Feng L, Long L and Li Y: The Ephrin-A5/EphA4 interaction modulates neurogenesis and angiogenesis by the p-Akt and p-ERK pathways in a mouse model of TLE. Mol Neurobiol 53: 561-576, 2016.

25. Kron MM, Zhang H and Parent JM: The developmental stage of dentate granule cells dictates their contribution to seizure-induced plasticity. J Neurosci 30: 2051-2059, 2010.

26. Parent JM, Yu TW, Leibowitz RT, Geschwind DH, Sloviter RS and Lowenstein DH: Dentate granule cell neurogenesis is increased by seizures and contributes to aberrant network reorganization in the adult rat hippocampus. J Neurosci 17: 3727-3738, 1997.

27. Song C, Xu W, Zhang X, Wang S, Zhu G, Xiao T, Zhao M and Zhao C: CXCR4 antagonist AMD3100 suppresses the long-term abnormal structural changes of newborn neurons in the intraventricular kainic acid model of epilepsy. Mol Neurobiol 53: 1518-1532, 2016.

28. Kobow K, Jeske I, Hildebrandt M, Hauke J, Hahnen E, Buslei R, Buchfelder M, Weigel D, Stefan H, Kasper B, et al: Increased reelin promoter methylation is associated with granule cell dispersion in human temporal lobe epilepsy. J Neuropathol Exp Neurol 68: 356-364, 2009

29. Haas CA and Frotscher M: Reelin deficiency causes granule cell dispersion in epilepsy. Exp Brain Res 200: 141-149, 2010.

30. Michelucci R, Pulitano P, Di Bonaventura C, Binelli S, Luisi C, Pasini E, Striano S, Striano P, Coppola G, La Neve A, et al: The clinical phenotype of autosomal dominant lateral temporal lobe epilepsy related to reelin mutations. Epilepsy Behav 68: 103-107, 2017.

31. Baek ST, Copeland B, Yun EJ, Kwon SK, Guemez-Gamboa A, Schaffer AE, Kim S, Kang HC, Song S, Mathern GW, et al: An AKT3-FOXG1-reelin network underlies defective migration in human focal malformations of cortical development. Nat Med 21: 1445-1454, 2015.

32. Korn MJ, Mandle QJ and Parent JM: Conditional disabled-1 deletion in mice alters hippocampal neurogenesis and reduces seizure threshold. Front Neurosci 10: 63, 2016.

33. Dobrzanski P, Hunter K, Jones-Bolin S, Chang H, Robinson C, Pritchard S, Zhao H and Ruggeri B: Antiangiogenic and antitumor efficacy of EphA2 receptor antagonist. Cancer Res 64: 910-919, 2004

This work is licensed under a Creative Commons Attribution-NonCommercial-NoDerivatives 4.0 International (CC BY-NC-ND 4.0) License. 